\title{
New Directions in Networks and Systems Approaches to Cardiovascular Disease
}

\author{
Päivi Pajukanta
}

Published online: 8 January 2013

(C) Springer Science + Business Media New York 2013

\begin{abstract}
Cardiovascular disease (CVD) is a broad definition for diseases of the heart and blood vessels with high mortality and morbidity worldwide. Atherosclerosis and hypertension are the most common causes of CVD, and multiple factors confer the susceptibility. Some of the predisposing factors are modifiable such as diet, smoking, and exercise, whereas others, including age, sex, and genetic composition are non-modifiable. Interactions between the different factors also contribute to the outcome. Development of cardiovascular disease starts in childhood and gradually involves multiple organs, tissues, and cell types with different functions. The molecular mechanisms underlying CVD are estimated to involve hundreds of genes. To better address the highly complex architecture and multiple properties leading to CVD, networks and systems approaches combining information at genomic, transcriptomics, methylomics, proteomics, metabolomics, and phenome level are being developed, with the ultimate goal to elucidate the cascade of dynamic changes leading to CVD in humans. This review will discuss co-expression networks and systems approaches from cardiovascular genetics point of view.
\end{abstract}

Keywords Epigenomics - Metabolomics - Network · Personal medicine $\cdot$ Systems genetics $\cdot$ Transcriptomics

P. Pajukanta $(\square)$

Department of Human Genetics, David Geffen School

of Medicine at UCLA, Gonda Center, Room 6335B,

695 Charles E. Young Drive South, Los Angeles, CA

90095-7088, USA

e-mail: ppajukanta@mednet.ucla.edu

\author{
Abbreviations \\ ASE Allele specific expression \\ CVD Cardiovascular disease \\ CHD Coronary heart disease \\ GWAS Genome-wide association study \\ HDL-C High-density lipoprotein cholesterol \\ LDL-C Low-density lipoprotein cholesterol \\ PPI Protein-protein interaction \\ QTL Quantitative trait locus \\ SNP Single nucleotide polymorphism \\ TGs Triglycerides \\ T2D Type 2 diabetes \\ WGCNA Weighted gene co-expression network analysis
}

\section{Introduction}

The Human Genome Project [1], HapMap Project [2], and 1,000 Genomes Project [3] together with recent highthroughput approaches have enabled extensive genomic scale searches of DNA sequence variants contributing to cardiovascular disease (CVD). In genome-wide association studies (GWAS) of single traits, tens of thousands of cases and controls have been genotyped for hundreds of thousands of single nucleotide polymorphisms (SNPs) throughout the human genome to search for CVD-associated variants at the DNA level. GWAS have been highly successful in identifying novel common variants for CVD. For example, a recent extensive GWAS meta-analysis identified 13 new loci associated with 6-17\% increase in the risk for coronary heart disease (CHD) per allele [4•]. The study also supported the diverse patophysiology of CVD, because only three of the 13 new loci were associated with traditional CHD risk factors, such as serum lipid 
levels [4•]. Similarly, a previous extensive lipid GWAS meta-analysis identified 95 loci for serum lipid traits in $\sim 100,000$ European origin individuals [5]. Taken together, the common variants identified in cardiovascular GWAS exhibit small to moderate effects on CVD phenotypes and explain $\sim 10 \%$ of the entire phenotypic variance of which some $50 \%$ is estimated to be heritable (discussed in [6]). Similar results were obtained with other complex cardiovascular diseases, such as hypertension and intracranial aneurysms $[7,8]$.

A recent gene centric meta-analysis of 32 studies with 66,240 individuals of European ancestry genotyped some 50,000 SNPs covering about 2,000 candidate genes to further expand the sets of genes contributing to serum lipid levels [9•]. The authors demonstrated that this approach indeed resulted in the identification of lipid-related variants in previously unreported genes: Five genes were reported for HDL-C; five for LDL-C; seven for TC; and six for TGs, respectively. Furthermore, the explained phenotypic variance was comparable to that from a meta-analysis of GWAS data [9•], which implies that the gene-centric approach can further increase the understanding of heritability of plasma lipids.

Overall the GWAS results suggest that other sources of variation such as rare and low-frequency variants; genegene and gene-environment interactions, and above DNA factors such as epigenetic changes each explain part of the remaining variance. Furthermore, the fact that single variants and traits do not alone explain a large proportion of CVD clearly indicates that networks and systems approaches are needed to complement the single genetic variant analysis and better model the complex interactive properties involved in the development and etiology of CVD $[10 \bullet$. There are several different types of biological networks such as protein-protein interaction (PPI) networks, metabolic networks, and transcript regulatory networks (reviewed recently in 11). These biological networks represent the functional or physical connectivity among genes [11••], and thus help identify closely connected genes, interaction patterns, and enrichment in functional categories. Systems genetics is based on the principle that a complex system has intrinsic features and interactions that cannot be simply derived from the additive effects of the individual parts [10••] (Fig. 1). In this review, I will focus on recent studies utilizing co-expression networks and systems approaches to elucidate CVD genetics.

High-Throughput Sequencing Searches for Variation at the DNA and RNA Level

Some GWAS loci overlapped with the genes implicated for monogenic forms of CVD, suggesting that in general GWAS loci could be excellent targets for resequencing in order to identify novel disease causing rare variants [12]. Targeted

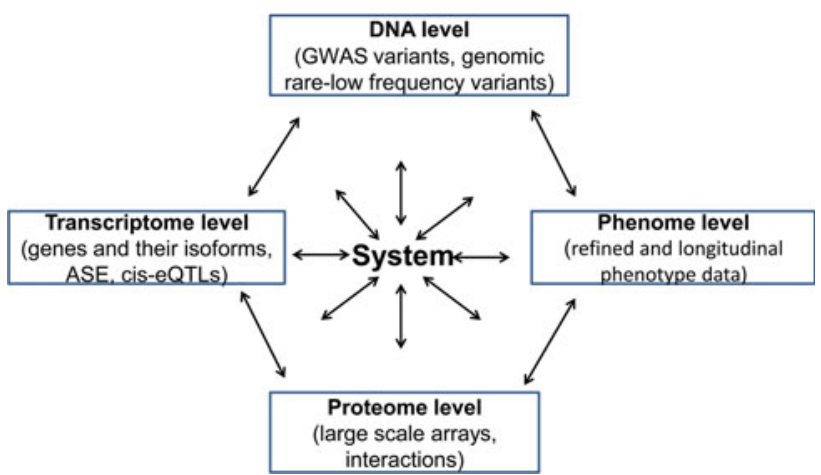

Fig. 1 Complex systems have intrinsic features and interactions that cannot be simply derived from the additive effects of the individual parts. ASE indicates allele specific expression

sequencing of GWAS loci has indeed identified rare coding variants with stronger functional effects, for example, in hypertriglyceridemic subjects [13]. Furthermore, exome sequencing and whole genome sequencing using efficient and large scale massive parallel sequencing approaches enable the identification of the full spectrum of variants, including rare and low frequency variants which may exhibit stronger effects than the common variants on CVD. Consequently, these variant types may also be easier to link to the underlying molecular mechanisms than common variants with subtle effects, especially when utilizing family-based sequencing in which co-segregation of the variants with the cardiovascular trait can be monitored and combined with quantitative trait locus (QTL) analysis, linkage analysis and/ or identity-by-descent sharing among the family members [14]. Exome sequencing has rapidly become the state-of-the art golden standard method in gene identification of monogenic forms of rare cardiovascular and other diseases [15], whereas its feasibility in unrelated individuals with complex common forms of CVD remains to be proven.

RNA sequencing provides a link from genome to transcriptome when investigating genetics of CVD $[10 \bullet \bullet, 16]$. It produces information not only about the variants of genes expressed in the particular tissue but also allows at an unprecedented resolution quantification of gene expression levels through the entire length of the transcript, characterization of known and novel splice forms, investigation of the currently largely unknown role of allele-specific expression in CVD, and joint analyses of these data for sequence variation and disease phenotypes [16]. A careful quality control is, however, essential in RNA sequencing to avoid sequencing errors due to lane, strand, and position biases (discussed in [17]).

Metabolomics Networks Identify Novel Genes for CVD

In genetic studies, it is crucial to investigate the phenotypes with the closest links to the genotypes. Man-made diagnostic 
criteria, cumulative disease endpoints and outcomes may not best reflect this critical genotype-phenotype link. Recent advances in measuring hundreds of metabolomics phenotypes including composition of different lipid particles from serum samples, for example, by nuclear magnetic resonance [18], have made it possible to search for both the heritability estimates of these parameters and their correlations with traditional lipid measurements, such as TGs, HDL-C, total cholesterol (TC), LDL-C, and the outcome, CHD. Reinvestigation of lipid GWAS data using heritable lipid metabolomics phenotypes measured by nuclear magnetic resonance have demonstrated how common SNPs explain more of the trait variance of lipid metabolomics phenotypes than of the serum composite lipid measurements, TGs, HDL-C, TC, and LDL-C [18].

A previous study introduced metabolomics networks as an approach to identify novel genes for atherosclerosis, combining existing GWAS data, metabolomics networks, and gene expression data [19••]. The authors investigated $\sim 6,600$ individuals with serum metabolomic profiles and GWAS data and identified 11 metabolic networks for a multivariate genome-wide association analysis using correlation structure of 130 metabolites. Overall, they detected 34 genome-wide significant loci, of which seven were new. When the authors compared these data to univariate tests, they noticed that multivariate association analysis identified almost twice as many significant associations as univariate tests [19••]. These data were also further supported by multi-tissue gene expression studies, suggesting that metabolomics networks help identify additional signals underlying CVD.

\section{Some GWAS Variants Regulate Transcription}

Systems genetics utilizes systems analysis of phenotypes and genetic variants at multiple levels [10••], and it has been an especially useful approach to follow-up variants identified in GWAS (reviewed in 11). For example, a sequence variant can influence expression of a gene nearby (cis-eQTL) or in a distant locus (trans-eQTL). As only a few of the common variants in the GWAS studies reside in the coding regions, many GWAS studies have explored whether the non-coding genome-wide significant variants have regulatory effects by investigating them in a cis-eQTL analysis of relevant tissues [12, 20]. Kathiresan et al. [12] identified several genome-wide significant lipid SNPs that altered liver expression of a regional gene not previously known to be involved in CVD. These observations were made in more than 900 human liver RNA samples, suggesting a mechanistic role for the novel variants and genes in a tissue highly relevant to lipid metabolism [12]. A recent study utilized DNase I sequencing to measure chromatin accessibility in 70 HapMap lymphoblastoid cell lines. The authors demonstrated that DNase I sensitivity QTLs (dsQTL) are important novel determinants of human expression variation as more than $50 \%$ of eQTL SNPs are also dsQTLs [21]. Yet, the major challenge of GWAS studies has been to convert the identified association signals to new functional knowledge and mechanistic insight. This is an inherent problem of a DNA-level genome-wide scan in which most of the screened common variants are either intronic or intergenic and often belong to extended LD intervals with multiple regional variants in tight LD.

A previous study utilized a systems genetics approach for the further investigation of the 48 genes implicated by GWAS for type 2 diabetes (T2D) in order to link GWAS variants to pathways and biological networks [22•]. The authors explored co-expression patterns of these 48 genes with genes expressed in human islets from 63 donors, of which nine had T2D. The authors identified gene co-expression and protein-protein interaction networks associated with islet insulin secretion and HbAlc. When they integrated the data to form a rank list of putative T2D genes, they observed that expression variation of the top 20 genes explained $24 \%$ of the variance in HbA1c. The study demonstrated how systems genetics can be utilized to connect T2D-associated SNPs to pathways relevant for human islet function and pathogenesis of T2D [22•].

\section{Transcript Networks Identify Novel Connections} Between Transcripts, Phenotypes, Variants, and Methylation Sites

Conventional differential gene expression analysis between two study groups suffers from correcting for multiple testing of thousands of transcripts. Few signals survive or exhibit strong enough fold changes between the tested groups, especially when investigating cases and controls of complex traits. Pathway analysis of the most differentially expressed lists of genes can partially circumvent this issue (reviewed recently in 11). In addition, gene set enrichment analysis (GSEA) has been widely used to identify enriched gene sets in transcriptomics [11, 23]. Transcript networks can also be built to explore transcript profiles from a systems perspective. Several methods have been developed for this purpose (reviewed recently in [10, 11]). One such method is the weighted gene co-expression network analysis (WGCNA) [24, 25••] that is designed to identify modules of densely interconnected genes by searching for genes with similar patterns of connectivity. The co-expression networks (modules) are constructed by correlation measures followed by hierarchical clustering and dynamic tree cut for module detection [24, 25••]. The gene co-expression modules summarize the main patterns of variation. The first principal component represents the summary of the module and is referred to as the module eigengene (ME). Thus, 


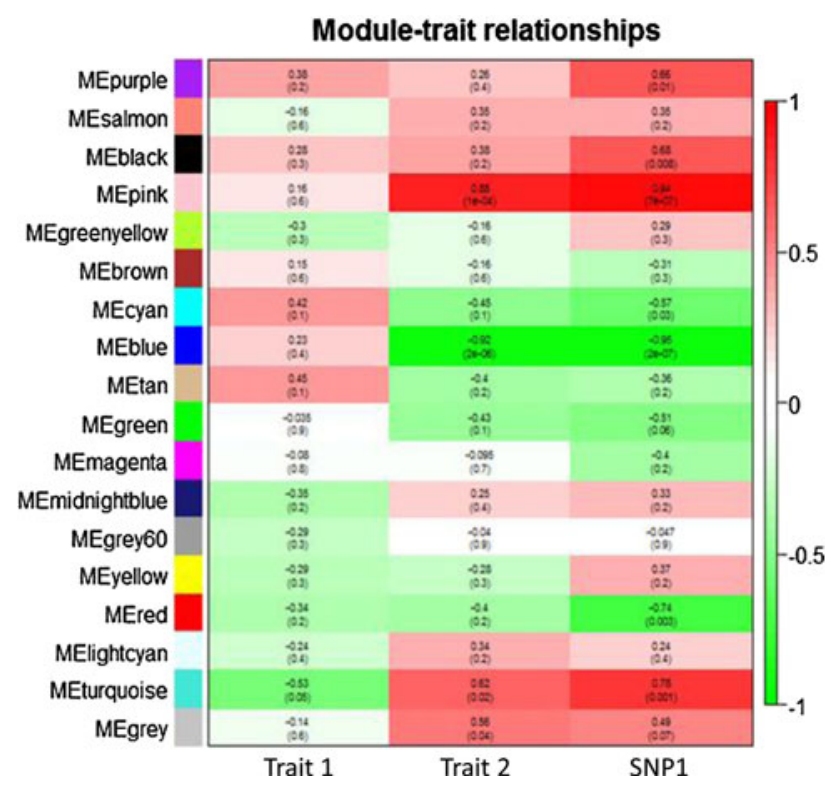

Fig. 2 Weighted Gene Co-expression Analysis (WGCNA) identifies co-expression modules that can be correlated with phenotypic traits and DNA variants. Correlations of module eigengenes (ME) are shown with two phenotypic traits and a SNP. The rows are labeled by the $M E$ color, and the columns are labeled by the clinical trait. The correlation coefficients are shown for each cell, and in parentheses is the $p$ value for the significance of the correlation

WGCNA can be utilized to identify gene co-expression modules summarizing the main trends in variation in the transcriptome data and subsequently the co-expression modules can be linked to clinical traits or binary outcomes and DNA/RNA variants (Fig. 2). Relating network modules instead of genes to a clinical trait is a major advantage of WGCNA. This module-based analysis will significantly reduce the burden of multiple testing to the number of modules and examined phenotypic traits instead of accounting for tens of thousands of multiple comparisons as is done in conventional differential expression analyses. Furthermore, the network modules that are significantly correlated with the disease status, phenotypic traits, and/or DNA/RNA sequence variants may subsequently be analyzed using pathway tools, such as DAVID [26, 27] to identify biologically meaningful functional enrichment categories. WGCNA also allows for investigation of module preservation between study samples and across tissues $[24,25 \bullet \cdot$. Furthermore, WGCNA can utilize gene expression data derived from both microarrays and RNA sequencing [28].

Recently WGCNA has also been utilized for genomic analysis of methylation data using the quantitative methylation intensity values (beta values) as estimates of methylation [29•]. These methylation intensity values are conceptually equivalent to the expression intensity since unmethylated promoters are associated with genes that are up-regulated and methylated promoters with genes that are down-regulated. Importantly, gene expression and methylation networks can be compared across tissues, and furthermore, the co-expression and co-methylation modules can be statistically correlated with phenotypic traits and sequence variant data. Thus, network approaches such as WGCNA allow combination of data at multiple levels, including phenotype, DNA variation, transcript and methylation data.

There are several examples of WGCNA analysis identifying transcript networks in animal and human studies [30-33]. In our own previous study, we constructed weighted gene co-expression networks in adipose RNA samples from Mexican familial combined hyperlipidmia (FCHL) family members who underwent a subcutaneous fat biopsy [33]. By integrating the upstream transcription factor 1 (USF1) SNP rs3737787 with the gene expression levels in adipose tissue, we were able to attribute function to this SNP in USF1 associated with FCHL in European origin and Mexican families [34, 35]. Our study demonstrated that the common USF1 SNP contributes to FCHL disease risk by modulating the expression of a group of genes functionally related to lipid metabolism.

\section{Towards Personalized Omics}

Networks and systems genetics approaches lay a foundation for personalized medicine that aims to combine biological high-throughput data at multiple levels through-out an individual's life. A recent study followed one individual over a 14-month period by collecting genomic, transcriptomic, proteomic, metabolomic, and autoantibody profiles from the individual to build an integrative personal omics profile (iPOP) [36॰]. Interestingly, the authors discovered extensive, dynamic changes in different molecular components and biological pathways across healthy and diseased conditions. Specifically, the authors detected extensive heteroallelic changes during healthy and diseased states and found 497 and 1,047 genes with allele-specific expression differences during the two viral infections caused by HRV and RSV the person endured. They also observed for example how glucose levels increased after the RSV infection for several months [36•], demonstrating thus for the first time a link between an RSV infection and the onset of T2D in human.

\section{Conclusions}

High-throughput data produced at the genome, transcriptome, proteome, metabolome, and phenome level enable network and systems-based analysis of CVD. Each level is important and refined detailed data is needed for a proper representation of a level, emphasizing especially the 
importance of careful clinical and metabolomics phenotyping. Recent systems-based follow-ups of GWAS variants using metabolomics and transcriptomics data have started to elucidate the usefulness of the network and systems approaches. Interpretation of the systems data is clearly more challenging than that of single trait and variant data, and there is also a high demand to develop rigorous follow-up and biology-based replication approaches for the network approaches. Despite these challenges, the network and systems approaches have a high potential to lead us towards personalized medicine of human beings.

Disclosure The author reported no potential conflict of interest relevant to this article.

\section{References}

Papers of particular interest, published recently, have been highlighted as:

- Of importance

- Of major importance

1. International Human Genome Sequencing Consortium. Finishing the euchromatic sequence of the human genome. Nature. 2004; 431:931-45.

2. The International HapMap Consortium. A haplotype map of the human genome. Nature. 2005;437:1299-320.

3. The 1000 Genomes Project Consortium. A map of human genome variation from population-scale sequencing. Nature. 2010; 467:1061-73.

4. - Schunkert H, König IR, Kathiresan S, et al. Large-scale association analysis identifies 13 new susceptibility loci for coronary artery disease. Nat Genet. 2011;43:333-8. This paper is an excellent example of a cardiovascular GWAS study.

5. Teslovich TM, Musunuru K, Smith AV, et al. Biological, clinical and population relevance of 95 loci for blood lipids. Nature. 2010;466:707-13.

6. Weissglas-Volkov D, Pajukanta P. Genetic causes of high and low serum HDL-cholesterol. J Lipid Res. 2010;51:2032-57. Review.

7. Ehret GB. Genome-wide association studies: contribution of genomics to understanding blood pressure and essential hypertension. Curr Hypertens Rep. 2010;12:17-25. Review.

8. Yasuno K, Bilguvar K, Bijlenga P, et al. Genome-wide association study of intracranial aneurysm identifies three new risk loci. Nat Genet. 2010;42:420-5.

9. - Asselbergs FW, Guo Y, van Iperen EP, et al. Large-Scale GeneCentric Meta-analysis across 32 Studies Identifies Multiple Lipid Loci. Am J Hum Genet. 2012; 91:823-38. http://dx.doi.org/10. 1016/j.ajhg.2012.08.032. This paper illustrates well the genecentric approach to identify CVD genes.

10. • MacLellan WR, Wang Y, Lusis AJ. Systems-based approaches to cardiovascular disease. Nat Rev Cardiol. 2012;9:172-84. This is an excellent recent review of systems-based approaches in CVD.

11. •• Sun YV. Integration of biological networks and pathways with genetic association studies. Hum Genet. 2012;131:1677-86. This is a detailed recent review of different types of networks and pathways approaches.

12. Kathiresan S, Willer CJ, Peloso GM, et al. Common variants at 30 loci contribute to polygenic dyslipidemia. Nat Genet. 2009;41: 56-65.
13. Johansen CT, Wang J, McIntyre AD, et al. Excess of rare variants in non-genome-wide association study candidate genes in patients with hypertriglyceridemia. Circ Cardiovasc Genet. 2012;5:66-72.

14. Reddy MV, Iatan I, Weissglas-Volkov D, et al. Exome sequencing identifies 2 rare variants for low high-density lipoprotein cholesterol in an extended family. Circ Cardiovasc Genet. 2012;5:538-46.

15. Ng SB, Buckingham KJ, Lee C, et al. Exome sequencing identifies the cause of a mendelian disorder. Nat Genet. 2010;42:30-5.

16. Pickrell JK, Marioni JC, Pai AA, et al. Understanding mechanisms underlying human gene expression variation with RNA sequencing. Nature. 2010;464:768-72.

17. Pickrell JK, Gilad Y, Pritchard JK. Comment on "Widespread RNA and DNA sequence differences in the human transcriptome”. Science. 2012;335:1302; author reply 1302.

18. Tukiainen T, Kettunen J, Kangas AJ, et al. Detailed metabolic and genetic characterization reveals new associations for 30 known lipid loci. Hum Mol Genet. 2012;21:1444-55.

19. •• Inouye M, Ripatti S, Kettunen J, et al. Novel Loci for metabolic networks and multi-tissue expression studies reveal genes for atherosclerosis. PLoS Genet. 2012; 8(8):e1002907. This is an interesting paper utilizing metabolomics networksto identify novel loci for atherosclerosis.

20. International Consortium for Blood Pressure Genome-Wide Association Studies. Genetic variants in novel pathways influence blood pressure and cardiovascular disease risk. Nature. 2011;478: 103-9.

21. Degner JF, Pai AA, Pique-Regi R, et al. DNase I sensitivity QTLs are a major determinant of human expression variation. Nature. $2012 ; 482: 390-4$.

22. - Taneera J, Lang S, Sharma A, et al. A systems genetics approach identifies genes and pathways for type 2 diabetes in human islets. Cell Metab. 2012;16:122-34. The paper follows up T2D GWAS genes using co-expression and protein-protein interaction approaches in human islets.

23. Subramanian A, Tamayo P, Mootha VK, et al. Gene set enrichment analysis: a knowledge based approach for interpreting genome-wide expression. Proc Natl Acad Sci USA. 2005;102: 15545-50.

24. Zhang B, Horvath S. A general framework for weighted gene coexpression network analysis. Stat Appl Genet Mol Biol. 2005; 4:Article17.

25. • Langfelder P, Horvath S. WGCNA: an R package for weighted correlation network analysis. BMC Bioinformatics. 2008; 9:559. This paper introduces the weighted gene co-expression analysis.

26. Huang DW, Sherman BT, Lempicki RA. Systematic and integrative analysis of large gene lists using DAVID Bioinformatics Resources. Nature Protoc. 2009;4:44-57.

27. Huang DW, Sherman BT, Lempicki RA. Bioinformatics enrichment tools: paths toward the comprehensive functional analysis of large gene lists. Nucleic Acids Res. 2009, 37:1-13.

28. Haas BE, Horvath S, Pietiläinen $\mathrm{KH}$, et al. Adipose Co-expression networks across Finns and Mexicans identify novel triglyceride-associated genes. BMC Med Genomics. 2012;5:61.

29. - van Eijk KR, de Jong S, Boks MP, et al. Genetic analysis of DNA methylation and gene expression levels in whole blood of healthy human subjects. BMC Genomics. 2012;13:636. This paper utilizes WGCNA to build methylation networks.

30. Voineagu I, Wang X, Johnston P, et al. Transcriptomic analysis of autistic brain reveals convergent molecular pathology. Nature. 2011;474:380-4.

31. Fuller TF, Ghazalpour A, Aten JE, et al. Weighted gene coexpression network analysis strategies applied to mouse weight. Mamm Genome. 2007;18:463-72.

32. Oldham MC, Horvath S, Geschwind DH. Conservation and evolution of gene coexpression networks in human and 
chimpanzee brains. Proc Natl Acad Sci U S A. 2006;21(103): 17973-78.

33. Plaisier CL, Horvath S, Huertas-Vazquez A, et al. A systems genetics approach implicates USF1, FADS3, and other causal candidate genes for familial combined hyperlipidemia. PLoS Genet. 2009;5(9):e1000642.

34. Pajukanta P, Lilja HE, Sinsheimer J, et al. Familial combined hyperlipidemia is associated with upstream transcription factor 1 (USF1). Nat Genet. 2004;36:371-6.
35. Huertas-Vazquez A, Aguilar-Salinas C, Lusis AJ, et al. Familial combined Hyperlipidemia in Mexicans: association with upstream transcription factor 1 and linkage on chromosome 16q24.1. Arterioscler Thromb Vasc Biol. 2005;25:1985-91.

36. - Chen R, Mias GI, Li-Pook-Than J, et al. Personal omics profiling reveals dynamic molecular and medical phenotypes. Cell. 2012;148:1293-307. This paper introduces the idea of personal omics profiling utilizing high-throughput data at multiple levels. 